\title{
Y A-T-Il Une Relation Entre Teneur En Chlorophylle Et Accumulation De La Proline Chez Les Céréales À Paille Sous Déficit Hydrique?
}

\author{
Merabta Sarra (PhD Student) \\ Zerafa Chafia (PhD Student) \\ Benlaribi Mostefa (Prof.)
}

Laboratoire de Développement et Valorisation des Ressources

Phytogénétiques, Université des Frères Mentouri Constantine 1, Algérie

Doi: 10.19044/esj.2018.v14n15p234 URL:http://dx.doi.org/10.19044/esj.2018.v14n15p234

\begin{abstract}
This paper focuses on the relationship between two genotypes of durum wheat (Triticum durum Desf.): Gamgoum Rekham (GGR) and Haurani; two genotypes of common wheat (Triticum aestivum L.): Florence aurore 8193 (FA) and Mexipak; and two genotypes of barley (Hordeum vulgare L.): Manel and Saïda 183. These genotypes were subjected to a water deficit during a period of twenty days at the heading stage. The proline content and the chlorophyll content are determined on the standard leaves both on the control and on the sample subjected to water stress after twenty days and after one and two weeks, following the return of watering. Despite the fact that these varieties are cultivated under the same conditions on the Algerian highlands, their reactions to the test conditions are very different. As a matter of fact, GGR and FA recorded very high levels of proline at the end of the stress. This, however, was at the moment when the two barley genotypes presented relatively low values for the two studied parameters. After the return of watering, the proline contents at the various genotypes returned gradually to those of the controls. The GGR genotype recovers more quickly than the others. As for the chlorophyll content, it evolves, unevenly, in the course of the test, showing no evolutionary indication in relation to the proline.
\end{abstract}

Keywords: Hydrous deficit, heading, proline, chlorophyl, return of watering

\section{Résumé}

L'étude que nous proposons a porté sur deux génotypes de blé dur (Triticum durum Desf.): Gamgoum Rekham (GGR) et Haurani ; deux génotypes de blé tendre (Triticum aestivum L.) : Florence aurore 8193 (FA) et Mexipak et deux génotypes d'orge (Hordeum vulgare L.) : Manel et Saïda 
183. Ces génotypes sont soumis à un déficit hydrique pendant une période de vingt jours $(20 \mathrm{j})$ au stade d'épiaison. La teneur en proline et le contenu en chlorophylle sont déterminés sur les feuilles étendards aussi bien sur le témoin que sur l'échantillon soumis à la contrainte hydrique après vingt jours et après une et deux semaines, suite au retour de l'arrosage. En dépit du fait que ces variétés sont cultivées dans les mêmes conditions sur les hauts plateaux algériens, leurs réactions aux conditions d'essai s'avèrent très différentes. En effet, GGR et FA enregistrent des teneurs en proline très élevées à l'issue du stress, au moment où les deux génotypes d'orge présentent des valeurs relativement faibles pour les deux paramètres étudiés. Après retour de l'arrosage les teneurs en proline chez les différents génotypes reviennent progressivement vers celles des témoins ; le génotype GGR récupère plus rapidement que les autres. Quant à la teneur en chlorophylle, elle évolue, en dents de scie, au cours de l'essai, ne présentant pas d'indication évolutive par rapport à la proline.

Mots-clés : Déficit hydrique, épiaison, proline, chlorophylle, retour de l'arrosage

\section{Introduction}

En conditions pluviales, la culture des céréales pratiquée sur de grandes étendues au même titre que les autres grandes cultures est exposée annuellement soit à l'insuffisance des précipitations, soit à la mauvaise répartition de ces précipitations dans le temps le long du cycle biologique de la plante (Benlaribi et al., 2014). La sécheresse en Algérie et dans d'autres pays du Maghreb est largement reconnue comme le premier facteur limitant de la production agricole et constitue une contrainte permanente. La lutte contre ce facteur climatique doit être envisagée à travers une approche multidisciplinaire (Monneuveux, 1991 ; Benlaribi \& Monneuveux, 1988) qui intègre essentiellement, dans ce cas , l'irrigation d'appoint, les techniques culturales et la sélection d'un matériel végétal adapté.

Le déficit hydrique provoque chez les plantes la mise en place d'un état de régulation grâce à une accumulation de composés osmo-régulateurs. Parmi ces derniers les acides aminés libres peuvent jouer un rôle significatif (Jones et al., 1980) à côté des ions minéraux $\underline{\mathrm{NO}}_{3}^{-}, \mathrm{K}^{+}, \mathrm{Cl}^{-}$(Salsac \& Monneuveux, 1991).

L'accumulation de la proline, acide iminé, notamment dans les feuilles de plantes sous contrainte hydrique, est décrite depuis fort longtemps déjà (Palfi et al., 1973 ; Hanson et al.,1977 ; Tan \& Halloran, 1982). En effet, de nombreux travaux ont révélé une augmentation de la quantité de proline dans les tissus foliaires par rapport au contenu dans les échantillons témoins (Benlaribi \& Monneuveux, 1988 ; Zerafa, 2006 ; et Benlaribi et al., 2014). 
Cette molécule est considérée comme un indicateur aussi bien des stress biotiques (Mohanty \& Sridhar, 1982) que des stress provoqués par des contraintes abiotiques tel que la salinité, le déficit hydrique (Hubac et al., 1980). Étant de l'ordre de $2 \mu$ moles.mg-1 MS (masse de substance sèche) dans les conditions d'alimentation hydrique non limitante, la teneur en proline peut atteindre des valeurs beaucoup plus élevées de l'ordre de $45 \mu$ moles.mg-1 MS, soit près de 25 fois la valeur initiale voire d'avantage selon le degré de sévérité du déficit hydrique développé (Benlaribi \& Chaib, 1995).

L'augmentation de la teneur foliaire en proline libre a été considérée pendant longtemps comme le résultat du seul phénomène de protéolyse. Mc Michael et Elmore (1977) observent toutefois une réduction de la teneur en acide glutamique concomitante de l'augmentation de la teneur en proline et suggèrent que l'accumulation de la proline est due à une synthèse accrue à partir de l'acide glutamique. Alors que pour (Hubac et al., 1980) l'accumulation résulterait d'un double phénomène d'hydrolyse et de synthèse. Ainsi, ce métabolite primaire dont la synthèse et la régulation sont encore peu maitrisées est induit sous l'effet de contraintes de l'environnement de culture et notamment sous l'effet d'une alimentation hydrique contraignante.

Il est à remarquer que Hubac (1967) et le Saint (1969) ont indiqué que la proline exogène appliquée sur des plantules entraine une augmentation de leur résistance à la sécheresse. Par ailleurs, Tahri et al. (1997) étudiant l'effet d'un stress osmotique au PEG sur trois variétés de blé dur obtient une accumulation de la proline dans les feuilles. En revanche, ils ont enregistré une diminution de leurs teneurs en pigments chlorophylliens expliquant le phénomène par une baisse de leur activité glutamine synthétase, enzyme impliquée dans la biosynthèse du glutamate, précurseur commun à la proline (Weinman \& Mehul, 2004) et aux pigments chlorophylliens.

Le rapport chlorophylle $(\mathrm{a} / \mathrm{b})$ est un bon indicateur du seuil de tolérance au stress hydrique, la chlorophylle constituant la base des systèmes photosynthétiques par le captage de l'énergie solaire. De plus, elle contient par le biais des chloroplastes des enzymes qui sont des catalyseurs et qui rendent ainsi possible les réactions chimiques des différentes étapes de la photosynthèse (Hopkins, 2003).

La tolérance remarquable de l'appareil photosynthétique à la déshydratation suggère que la survie des plantes en manque d'eau est en partie due à l'entretien de la capacité photosynthétique des feuilles, permettant le rétablissement rapide des plantes suite à une période de déficit hydrique (Bouras, 2001).

L'objectif de la présente étude est de contribuer à préciser la nature de la relation, s'il y en a une, entre les deux paramètres étudiés, à savoir la proline et la chlorophylle. 


\section{Matériels et méthodes}

\section{Matériel végétal}

L'étude a porté sur six variétés de céréales à consommation humaine d'origine locale et d'introduction : Gamgoum Rekham (GGR) et Haurani de blé dur (Triticum durum Desf), Florence aurore 8193 (FA) et Mexipak de blé tendre (Triticum aestivum L.) et Manel et Saïda 183 d'orge (Hordeum vulgare L.) (Tableau I).

Tableau I. Génotypes étudiés et leur origine.

\begin{tabular}{|c|c|c|}
\hline Genre & Nom du génotype & Son origine \\
\hline \multirow{2}{*}{ Blé dur } & Gamgoum Rekham (GGR) & I.T.G.C Tiaret Algérie \\
\cline { 2 - 3 } & Haurani & Syrie/Liban \\
\hline \multirow{2}{*}{ Blé tendre } & Florence aurore 8193(FA). & Tunisie-Algérie. \\
\cline { 2 - 3 } & Mexipak. & Mexique, Pakistan1973 \\
\hline \multirow{2}{*}{ Orge } & Manel. & Tunisie. \\
\cline { 2 - 3 } & Saïda 183. & Algérie. \\
\hline
\end{tabular}

\section{Conditions expérimentales}

Le semis est réalisé manuellement dans des pots de $19 \times 26 \times 18 \mathrm{~cm}$ contenant un sol agricole (argilo-limoneux), caractérisé par une capacité au champ relativement élevée (41\%). La densité de semis est de 14 grains / pot.

Les pots sont installés dans une serre vitrée selon un dispositif en bloc de Fisher randomisé à raison de trois répétitions pour chaque variété sous les conditions semi-contrôlées suivantes : (photopériode 15h, éclairement naturel, température $9^{\circ}-15^{\circ} \mathrm{C}$ la nuit, $17^{\circ}-28^{\circ} \mathrm{C}$ le matin et $20^{\circ}-45^{\circ}$ l'après-midi, humidité relative $60 \%$ ) comme indiqué sur les deux graphiques de la (Figure 1). Le développement des plantules se poursuit et l'arrosage est entrepris régulièrement à raison de $300 \mathrm{~mm}$ d'eau trois fois par semaine.

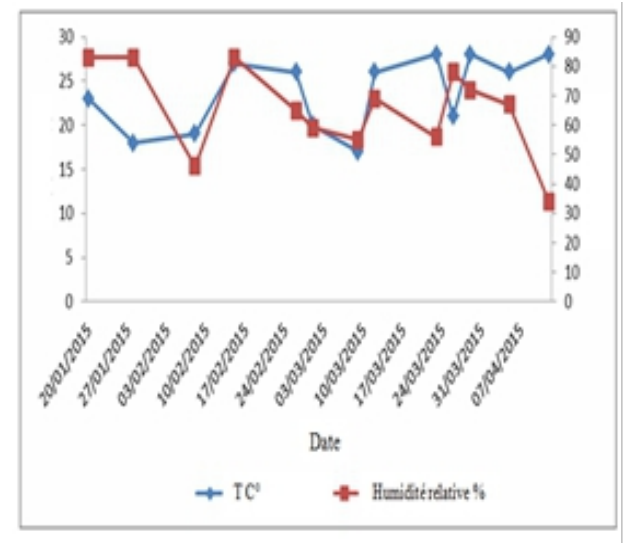

Le matin.

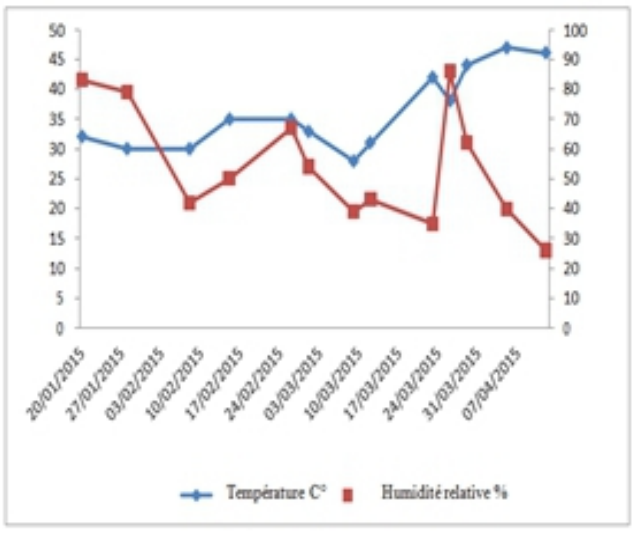

L'après-midi.

Figure 1. Variation de la température ambiante $\left(\mathrm{TC}^{\circ}\right)$ et de l'humidité relative $\%$ au niveau de la serre durant l'expérience. 


\section{Application du stress hydrique}

La première répétition des six génotypes utilisés est arrosée normalement et les plantes sont considérées comme témoins sans déficit hydrique (SDH) à $80 \%$ de la capacité au champ (CC) durant tout le long de notre étude, cependant les deux rangées restantes ont subi un stress (restriction) hydrique de 20 jours par arrêt d'arrosage durant la phase d'épiaison du cycle de développement (avec déficit hydrique ADH). Des échantillons foliaires (feuilles étendards) des plantes témoins et stressées sont utilisés pour des analyses au laboratoire à raison de trois répétitions par variété pour les deux traitements (SDH) et (ADH).

Après les analyses ces mêmes deux rangées (2 et 3 ) seront ré-arrosées de nouveau avec la quantité habituelle d'eau, et les échantillons sont prélevés après : $24 \mathrm{~h}$ (après retour de l'arrosage 1, APR1) et après une semaine (après retour de l'arrosage 2, APR2) pour l'étude du comportement biochimique après la réhydratation comme pour les deux traitements précédent (SDH et $\mathrm{ADH})$.

\section{Méthodes de mesures}

*La proline facilement oxydée par la ninhydrine (Larbier \& Leclercq, 1992) est dosée sur le tiers médian de la feuille étendard par la méthode de Troll et Lindsley (1955) modifiée par Dreier et Goring (1974). Pour la séparation des phases, le benzène toxique a été remplacé par le toluène conformément aux travaux de Hanson et al. (1977). La proline est alors évaluée par spéctrophotométrie sous une longueur d'onde de $528 \mathrm{~nm}$. Le poids de la matière sèche des différents échantillons est déterminé après passage à l'étuve $\left(80^{\circ} \mathrm{C}\right.$ pendant 24 heures $)$ des résidus de l'extraction.

* La chlorophylle a et b est déterminée grâce à la méthode de Mac Kiney (1941) et Arnon (1949) simplifiée par (Francis et al., 1970) qui utilise une solution d'acétone et d'éthanol. La densité optique est déterminée à $663 \mathrm{~nm}$ pour la chlorophylle a et à 645 pour la chlorophylle $b$.

*Les résultats obtenus au cours de cette expérimentation correspondent à la moyenne de trois répétitions et ils sont testés par une analyse de la variance à deux facteurs, réalisée à l'aide du logiciel XL-Stat. Le test de Newman-Keuls a permis d'exprimer les moyennes et de donner le seuil de signification.

\section{Résultats et Discussion}

Les teneurs en proline et les variations du contenu en chlorophylle totale sont représentées dans les figures 2,3 et 4 suivantes où l'on peut observer une nette augmentation de la teneur en proline des échantillons stressés par rapport aux échantillons témoins chez les variétés des trois espèces étudiées. 


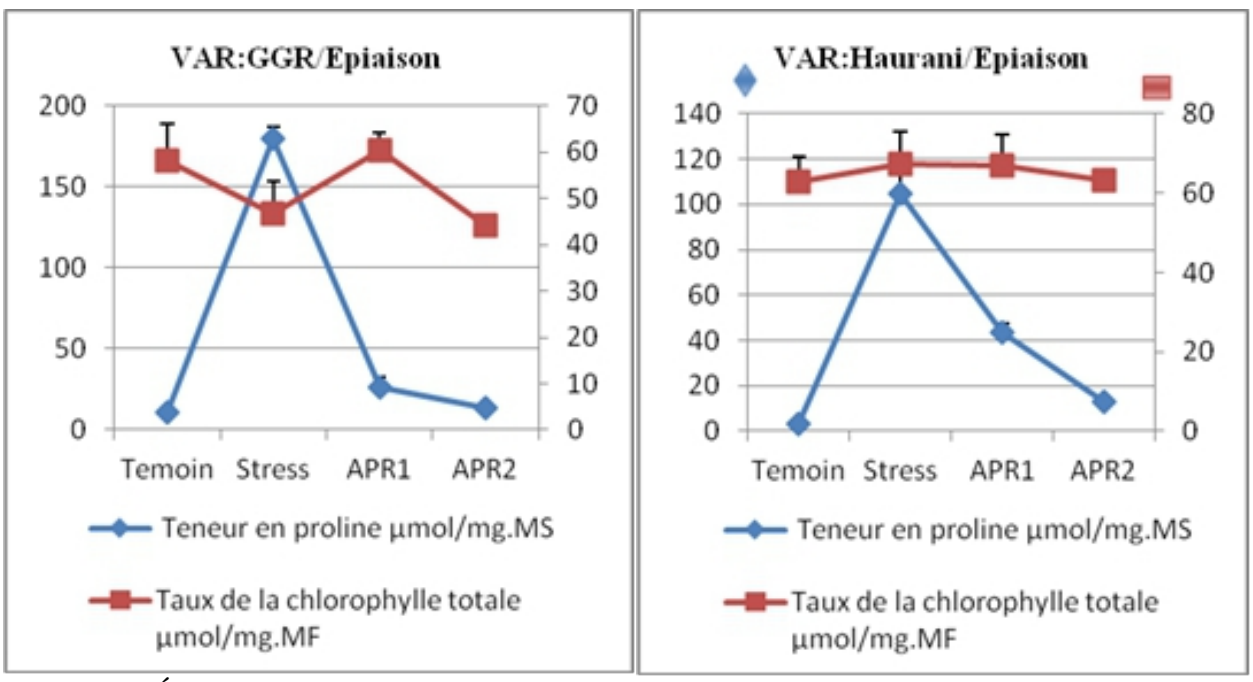

Figure 2. Évolution de la teneur en proline et du contenu en chlorophylle chez les deux variétés de blé dur.

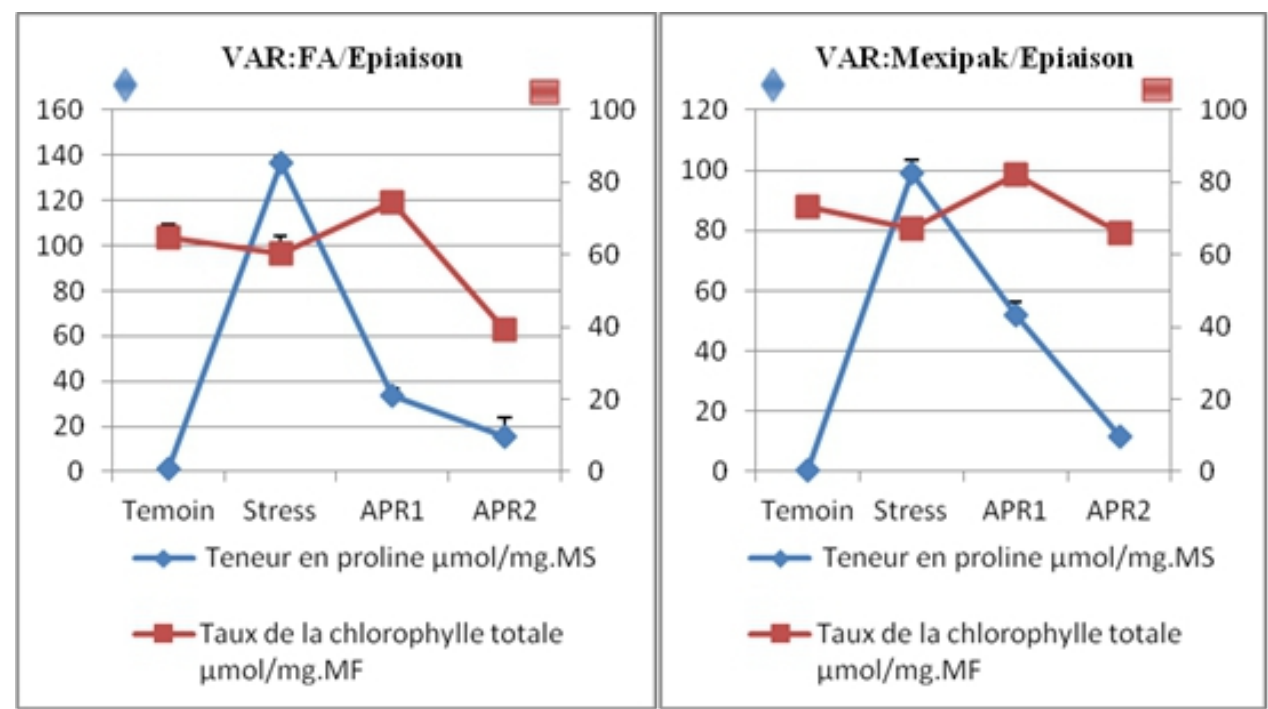

Figure 3. Évolution de la teneur en proline et du contenu en chlorophylle chez les deux variétés de blé tendre. 


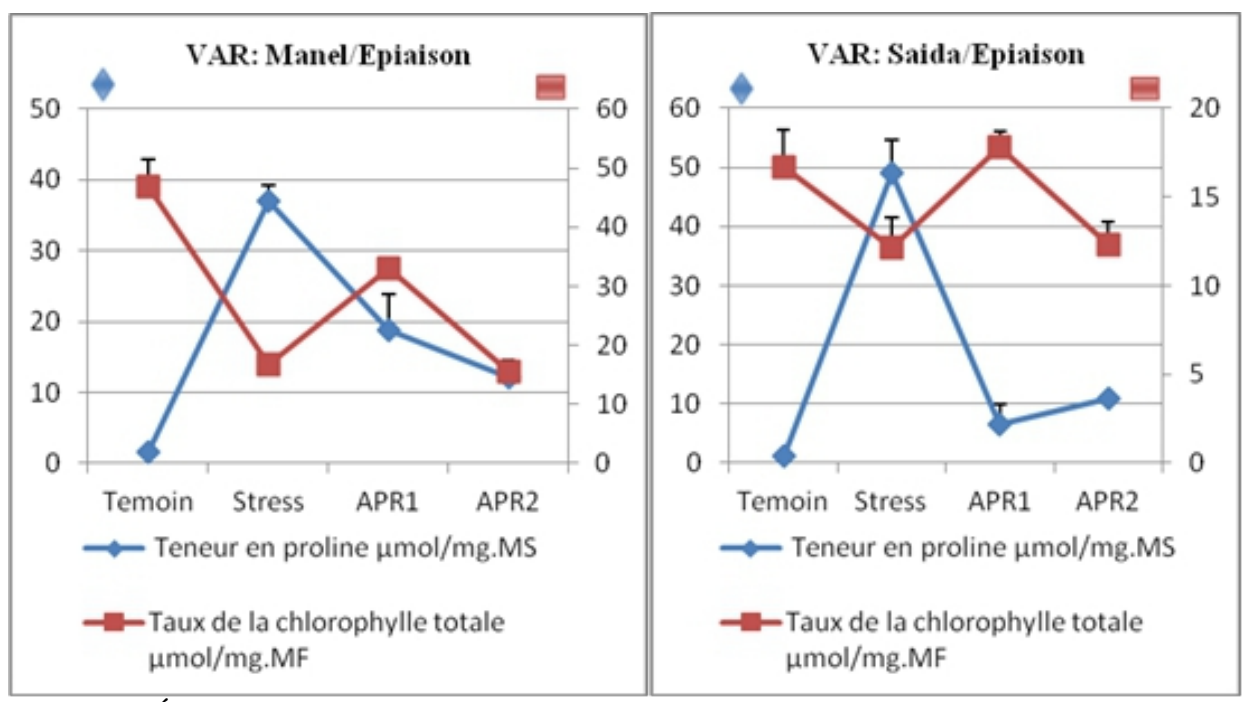

Figure 4. Évolution de la teneur en proline et du contenu en chlorophylle chez les deux variétés d'orge.

En ce qui concerne l'accumulation de la proline (Tableau II), l'observation globale des résultats dégagés par les trois espèces lors du déficit hydrique place la variété de blé dur GGR en premier, suivie par la variété de blé tendre FA ; arrivent ensuite les variétés Haurani et Mexipak et enfin les deux variétés d'orge Manel et Saïda 183.

On remarque une diversité dans le comportement par rapport à l'accumulation de ce composé aminé aussi bien lors des ADH qu'au moment de la reprise de l'arrosage.

Tableau II. Moyennes de la teneur en proline dans les quatre traitements hydriques.

\begin{tabular}{|c|c|c|c|c|c|}
\cline { 2 - 6 } \multicolumn{1}{c|}{} & \multicolumn{2}{c|}{ Traitements } & $(\mu \mathrm{mol} / \mathrm{mg} . \mathrm{MS})$ \\
\cline { 2 - 6 } & Variété & SDH & ADH & APR1 & APR2 \\
\hline \multirow{3}{*}{ Blé dur } & GGR & $11,03 \pm 0,50$ & $180,06 \pm 7,10$ & $26,14 \pm 6,70$ & $13,80 \pm 2,06$ \\
\cline { 2 - 6 } & Haurani & $3,29 \pm 0,23$ & $104,79 \pm 9,56$ & $43,53 \pm 4,08$ & $13,29 \pm 0,80$ \\
\hline \multirow{3}{*}{ Blé tendre } & FA & $1,00 \pm 0,60$ & $136,67 \pm 2,38$ & $33,44 \pm 3,73$ & $15,29 \pm 8,45$ \\
\cline { 2 - 6 } & Mexipak & $0,59 \pm 0,27$ & $98,99 \pm 4,38$ & $51,86 \pm 4,65$ & $11,58 \pm 1,23$ \\
\hline \multirow{3}{*}{ Orge } & Manel & $1,52 \pm 0,26$ & $37,04 \pm 2,30$ & $18,85 \pm 4,97$ & $12,19 \pm 2,46$ \\
\cline { 2 - 6 } & Saïda 183 & $1,11 \pm 0,38$ & $49,15 \pm 5,45$ & $6,49 \pm 3,41$ & $10,90 \pm 0,57$ \\
\hline
\end{tabular}

La résorption de ce composé aminé après le $1^{\text {er }}$ réarrosage est en faveur de la variété d'orge Saïda 183 et Manel ; arrive ensuite la variété GGR suivie par FA, Haurani et enfin Mexipak (Figure 5). 
Après le deuxième réarrosage (APR2) tous les génotypes ont enregistré des valeurs faibles et voisines. La variété Saïda 183 a présenté la valeur la plus basse et FA la plus haute.

Cependant, ces teneurs demeurent encore relativement élevées par rapport à celles des témoins (SDH), à l'exception de GGR qui a enregistré une valeur témoin élevée et inexpliquée pour le moment (Annexe I).

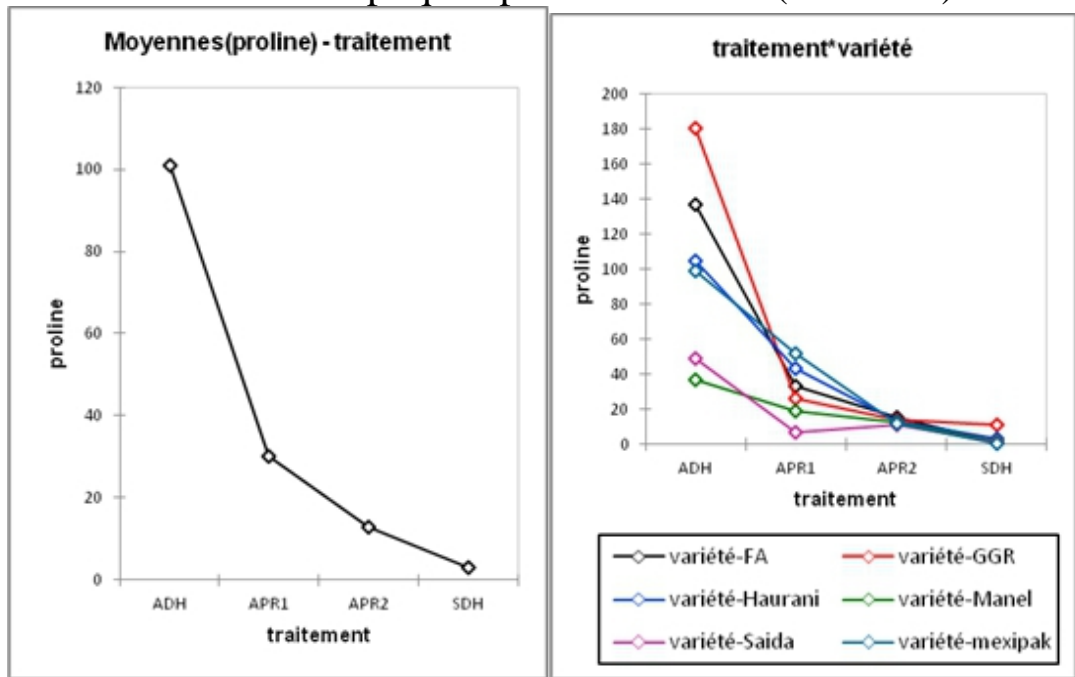

Figure 5. Courbe des moyennes des différents traitements pour les six génotypes.

Annexe I. Synthèse des comparaisons multiples par paires pour variété : Newman-Keuls (SNK) (Proline).

\begin{tabular}{cccccc}
\hline Modalité & Moyennes estimées(proline) & & Groupes & \\
\hline GGR & 57,759 & A & & & \\
FA & 46,599 & & B & & \\
Haurani & 41,225 & & C & \\
Mexipak & 40,753 & & C & \\
Manel & 17,401 & & D \\
Saida & 16,911 & & D \\
\hline
\end{tabular}

Les valeurs ainsi relevées dans l'étude sont en conformité avec celles présentées par de nombreux auteurs tels Chaïb et Benlaribi (2006), Rejaimia (2006), Zerafa (2006) et Benlaribi et al. (2014).

En effet, la plupart de ces auteurs ont constaté que l'accumulation de la proline dans les tissus foliaires des Triticum ne commence que lorsque la teneur en eau du sol avoisine $70 \%$ de la capacité au champ (C.C). Ce niveau d'humidité correspond approximativement à un potentiel hydrique foliaire de -10 à -12 bars et qu'en dessus de $70 \%$ de la C.C. le contenu en proline reste voisin de $2 \mu$ mole/mg.MS (Benlaribi \& Monneuveux, 1988).

Les teneurs en chlorophylle enregistrées dégagent deux tendances distinctes de génotypes: 
- les génotypes de blé dont les valeurs moyennes pour les quatre (04) traitements vont de 52,08 (GGR) à 72,10 $\mu$ moles/mg de matière fraîche (Mexipak).

- les deux génotypes d'orge dont les valeurs sont nettement inférieures et égales à 28,08 $\mu$ moles/mg.MF chez Manel et 14,71 chez Saïda 183 (Figure 6, Annexe II).

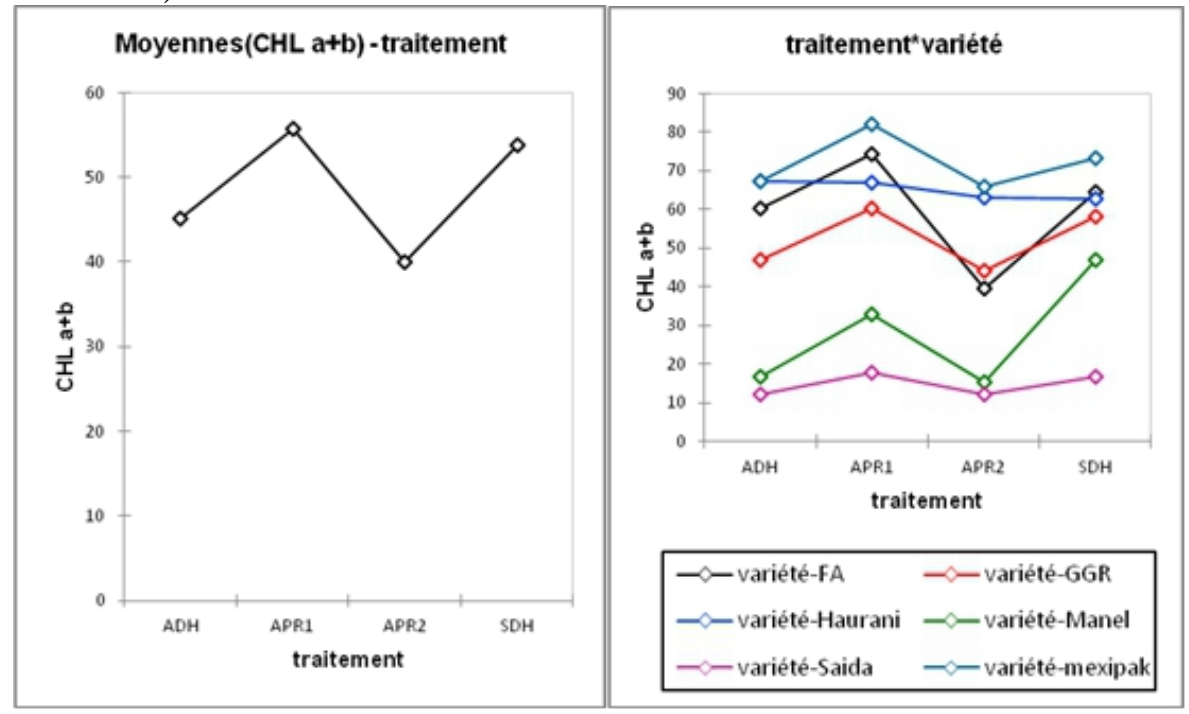

Figure 6. Courbe des moyennes des différents traitements pour les six génotypes.

Annexe II. Synthèse des comparaisons multiples par paires pour chaque variété : NewmanKeuls (SNK) (Chlorophylle).

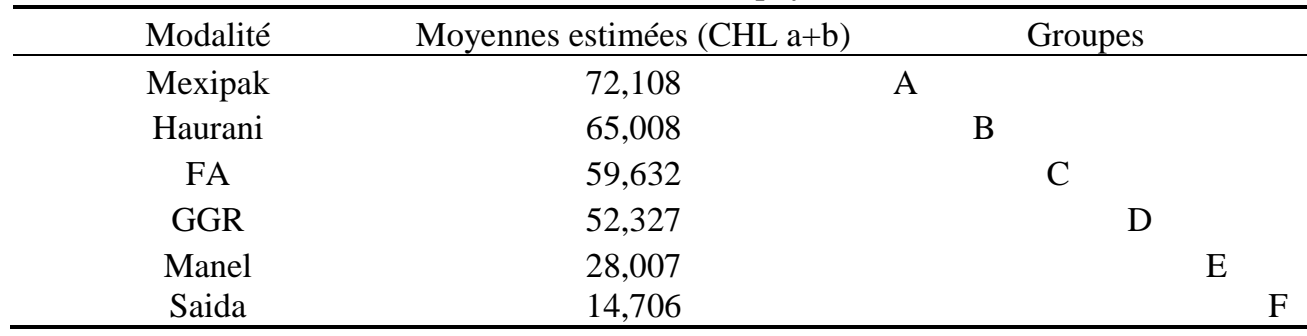

Ces valeurs de chlorophylle ne semblent pas obéir à l'évolution de la teneur en proline au cours du déficit hydrique et lors de la reprise de l'arrosage (Tableau III).

Tableau III. Moyennes du contenu en chlorophylle totale dans les quatre traitements hydriques.

\begin{tabular}{|c|c|c|c|c|c|}
\cline { 3 - 6 } \multicolumn{1}{c|}{} & \multicolumn{5}{c|}{ Traitements $(\mu \mathrm{mol} / \mathrm{mg} . \mathrm{MF})$} \\
\cline { 2 - 6 } \multicolumn{1}{c|}{ Blé dur } & Variété & SDH & ADH & APR1 & APR2 \\
\hline & GGR & $58,17 \pm 7,87$ & $46,75 \pm 7,07$ & $60,24 \pm 4,00$ & $44,15 \pm 2,17$ \\
\cline { 2 - 6 } & Haurani & $62,78 \pm 6,36$ & $67,29 \pm 8,31$ & $66,93 \pm 7,84$ & $63,04 \pm 1,79$ \\
\hline & FA & $64,62 \pm 3,70$ & $60,28 \pm 5,00$ & $74,24 \pm 2,71$ & $39,38 \pm 2,38$ \\
\hline
\end{tabular}




\begin{tabular}{|c|c|c|c|c|c|}
\hline Blé tendre & & & & & \\
\hline & Mexipak & $73,20 \pm 2,86$ & $67,32 \pm 1,34$ & $82,07 \pm 0,84$ & $65,84 \pm 1,84$ \\
\hline \multirow{2}{*}{ Orge } & Manel & $46,91 \pm 4,63$ & $16,71 \pm 1,29$ & $32,94 \pm 0,77$ & $15,47 \pm 1,46$ \\
\cline { 2 - 6 } & Saïda 183 & $16,68 \pm 2,12$ & $12,11 \pm 1,73$ & $17,78 \pm 0,95$ & $12,26 \pm 1,32$ \\
\hline
\end{tabular}

De cette manière, nos résultats ne suggèrent aucune relation entre l'évolution de la proline et celle de la chlorophylle pendant la période d'essai.

Ils sont donc en désaccord avec les conclusions avancées (présentées) par Tahri et al., (1997). Par conséquent, les deux voies de synthèse de ces deux composés (Proline et chlorophylle) ne semblent donc pas être connectées entre-elles pour être en concurrence pour leur prétendu précurseur commun le glutamate, comme il a été rapporté par Bengston et al. (1978) et Reddy et Veeranjaneyulu (1991).

\section{Conclusion}

L'application d'un stress hydrique prolongé provoque une accumulation de la teneur en proline dans les feuilles des deux genres expérimentés par rapport à celle des témoins. Cependant, cette accumulation est en faveur du genre Triticum à côté du genre Hordeum avec une diversité remarquable au sein des différentes variétés.

Quant à la composition totale en chlorophylle des organes étudiés, elle évolue en dents de scie n'entrainant aucune tendance indicative lors des différents traitements.

Après le retour de l'arrosage ( $20 \mathrm{j}$ après), il est observé une diminution progressive de la teneur en proline (APR1 et APR2), particulièrement chez les deux génotypes d'orge, et aucun changement concernant les teneurs en chlorophylle.

Les résultats auxquels nous sommes parvenus ne suggèrent donc aucune relation entre l'évolution de la proline et celle de la chlorophylle comme il est proposé par certains auteurs.

\section{References:}

1. Arnon, DI. (1949). Copper enzymes in isolated chloroplasts.Plant. Physiol, 24: 1-15.

2. Benlaribi, M., Merghem, R., Zarafa, C., \& Chaib, G. (2014). Une molécule, un métabolite primaire de contraintes mésologiques: la proline. Revue des régions arides-Numéro spécial- $\mathrm{n}^{\circ} 35: 1129-1138$.

3. Benlaribi, M. \& Chaib, G. (1995). Is proline accumulation a character of tolerance to drought? Congrès inter-drought, Montpellier . proceeding, INRA, W-5.

4. Benlaribi, M. \& Monneveux, PH. (1988). Etude comparée du 
comportement, en situation de déficit hydrique, de deux variétés algériennes de blé dur (Triticum durum Desf.) adaptées à la sécheresse. C.R.Acad.Agric.Fr.,74(5),73-83.

5. Bengston, C., Klockare, B., Klockare, R., Larsson, S., \& Sundquist, C. (1978). The after effect of water stress on chlorophyll formation during greening and the level of abscisic acid and proline in dark grown wheat seedlings. Plant Physiol., 43, pp. 205-212.

6. Bouras, F.Z. (2001). Effet du stress hydrique sur les composantes du rendement de quelques génotypes de blé dur. Thèse de magister I.N.A. El harrach.84 :15-23.

7. Chaib, G. (1998). Teneur en proline chez les différents organes de blé dur (Triticum durum desf) : essai d'explication des conditions d'accumulation sous manque d'eau. Thèse de Magister. Université de Constantine, Algérie.120p.

8. Chaib, G. \& Benlaribi, M. (2006). Proline Accumulation in durum wheat (Triticum durum Desf.) under water deficit. Arab. Univ. J. Agric. Sci., Ain Schams Univ ,Cairo,14(1),235-247.

9. Dreier, W. \& Goring, M. (1974). Der einfluss hoher Salzkonzèntrationen auf Versciedene physiologische parameter Von Maiswurzeln. Wiss. Z. der H.U. Berlin, Nath. Naturwiss r., 23:641644.

10. Francis, GW., Hertzberg, S., Andersen, K., \& Liaaen-Jensen, S. (1970) New carotenoid glycosides from Oscillatoria limosa. Phytochemistry 9:629-635

11. Hanson, AD., Nelsen, CE., \& Everson, EH. (1977). Evaluation of free proline accumulation as an index of drought resistance using two contrasting barley cultivars. Crop Science, 17,720-726.

12. Hopkins, W.G. (2003). Physiologie végétale, traduction de la $2^{\text {ème }}$ édition américaine, De Boeck Université, Bioénergétique et réactions photochimiques de la photosynthèse, 9, 163 -186

13. Hubac, C., Vieira Da, Silva, J. (1980). Indicateurs métaboliques de contraintes mésologiques. Physiol veg. 18: 45-54.

14. Hubac, C. (1967). C. R. Hebd. Seances Acad. Sci., Ser.D264: 12861289.

15. Jones, MM., Osmond, CB., \& Turner, NC. (1980). Accumulation of solutes in sorghum and sunflower leaves in response to water deficits. Australian Journal of Plant Physiology 7: 193-205.

16. Larbier, M. \& Leclercq, B. (1992). Nutrition et alimentation des volailles. Edition QUAE. Inra 358 : 93-107.

17. Le Saint, AM. (1969). C.R.Acad.Sci. Paris 269:1423-1426.

18. Mac Kinney, G. (1941). Absorption of light by chlorophyll solution. J. Biol. Chem. 140 : 315-322. 
19. Malki, S., Chaib, G., \& Benlaribi, M. (2002). Contribution à l'étude de la biodiversité du blé (Triticum sp) par le test de la proline. Séminaire international : biologie et environnement Constantine le 20.21 et 22 Octobre .Algérie 11-13.

20. Mc Michael, BH. \& Elmore, CD. (1977). Proline accumulation in water -stressed cotton leaves. Crop Sci., 17: 905-908.

21. Mohanty, SK. \& Sridhar, R. (1982). Physiology of ricetungro virus disease: proline accumulation due to infection. Physiol. Plant, 56:8893.

22. Monneuveux, PH. (1991). Quelle stratégie pour l'amélioration génétique de la tolérance au déficit hydrique des céréales d'hiver. In :Chalbi et Demarley Y.Ed. L'amélioration des plantes pour l'adaptation aux milieux arides. Ed.AUPELF- UREFJhonLibbey.INSA- INRA ,165-186.

23. Palfi, G., Bito, M., \& Palfi, Z. (1973). Free proline and water deficit in plant tissues. Fiziol. Rast.,20,189-193.

24. Redjamia, L. (2006). Accumulation de la proline comme indice moléculaire de (Triticum durum Desf.). Thèse magister, Université Constantine, $82 \mathrm{p}$.

25. Reddy, P.S. \& Veeranjaneyulu, K. (1991). Proline metabolism in senescing leaves of horsgram (Macrotyloma uniflorumLam.). J. Plant. Physiol., 137, pp. 381-383.

26. Salsac, L. \& Monneveux, PH. (1991). Relation entre la nutrition minérale et la tolérance au déficit hydrique, 49-66. In. Acevedo E. Conesa A. P. Monneveux Ph. Srivastava J. P. (Eds) Physiologybreeding of winter cereals for stressed Mediterranean environment. Montpellier, France, 3-6 July 1989.Colloques INRA N 55.

27. Tahri, E., Belabed, A., \& Sadki, K. (1997). Effet d'un stress osmotique sur l'accumulation de proline, de chlorophylle et des ARNm codant pour la glutamine synthétase chez trois variétés de blé dur (Triticum durum Desf.). Bulletin de 1'Institut Scientifique. Rebat.21: 81 - 89 p.

28. Tan BH \& Halloran GM (1982). Variation and correlations of proline accumulation in spring wheat. Crop Science, 22, 459-463.

29. Troll, W. \& Lindsley, J. (1955). A photometric method for the determination of proline .j.Biol.Chem., 215: 655-660.

30. Weinman, S. \& Méhul, P. (2004). Toute la biochimie. Sciences Sup: Sciences de la vie, ISSN 1636-2217. Dunod, 452p.

31. Zerafa, C. (2006). L'accumulation de la proline en tant que test précoce d'adaptation au déficit hydrique et indicateur moléculaire de diversité chez Triticum aestivum L. Mémoire de Magister. Fac des sciences, S.N.V, université de Constantine,Algèrie. 125p. 